\title{
AN ENTIRE FUNCTION WHICH HAS WANDERING DOMAINS
}

\author{
I. N. BAKER
}

(Received 1 November 1974)

\begin{abstract}
Let $f(z)$ denote a rational or entire function of the complex variable $z$ and $f_{n}(z), n=1,2, \cdots$, the $n$-th iterate of $f$. Provided $f$ is not rational of order 0 or 1 , the set $\mathfrak{C}$ of those points where $\left\{f_{n}(z)\right\}$ forms a normal family is a proper subset of the plane and is invariant under the map $z \rightarrow f(z)$. A component $G$ of $\mathfrak{C}$ is a wandering domain of $f$ if $f_{k}(G) \cap f_{n}(G)=\varnothing$ for all $k \geqq 1, n \geqq 1, k \neq n$. The paper contains the construction of a transcendental entire function which has wandering domains.
\end{abstract}

The theory of the iteration of a rational or entire function $f(z)$ of the complex variable $z$ deals with the sequence of natural iterates $f_{n}(z)$ defined by

$$
f(z)=z, \quad f_{1}(z)=f(z), \quad f_{n+1}(z)=f_{1}\left(f_{n}(z)\right), \quad n=0,1,2, \cdots .
$$

In the theory developed by Fatou $(1919,1926)$ and Julia $(1918)$ an important part is played by the set $\mathfrak{i}=\mathfrak{r}(f)$ of these points of the complex plane where $\left\{f_{n}(z)\right\}$ is not a normal family. Unless $f(z)$ is a rational function of order 0 or 1 , (which we henceforth exclude) the set $\mathfrak{r}(f)$ is a non-empty perfect set, whose complement $\mathfrak{S}=\mathfrak{S}(f)$ consists of an at most countably infinite collection of (open) components $G_{i}$, each of which is a maximal domain of normality of $\left\{f_{n}\right\}$.

It is shown by Fatou $(1919,1926)$ that $\mathfrak{f}(f)$ is completely invariant under the mapping $z \rightarrow f(z)$, i.e. if $\alpha$ belongs to $i(f)$ then so do $f(\alpha)$ and every solution $\beta$ of $f(\beta)=\alpha$. It follows that $\mathfrak{S}(f)$ is also completely invariant and, in particular, for each component $G_{i}$ of $\mathfrak{S}(f)$ there is just one component $G_{i}$ such that $f\left(G_{i}\right) \subset G_{i}$. By definition, the component $G_{0}$ of $\mathfrak{E}(f)$ is a wandering domain of $f$ if

$$
f_{k}\left(G_{0}\right) \cap f_{n}\left(G_{0}\right)=\varnothing \quad \text { for all } \quad 1 \leqq k, n<\infty, k \neq n .
$$

No examples of wandering domains for either entire or rational functions seem to be known and indeed Jacobson (1969) raises the question whether they can occur at all for rational $f$. Pelles also discusses the notion.

In Baker (1963) an entire function $g(z)$ was constructed as follows: 
Let $C=(4 e)^{-1}$ and $\gamma_{1}>4 e$. Then define inductively

$$
\gamma_{n+1}=C \gamma_{n}^{2}\left(1+\frac{\gamma_{n}}{\gamma_{1}}\right)\left(1+\frac{\gamma_{n}}{\gamma_{2}}\right) \cdots\left(1+\frac{\gamma_{n}}{\gamma_{n}}\right), \quad n=1,2, \cdots .
$$

Then $1<\gamma_{1}<\gamma_{2}<\cdots$ and [c.f. Baker (1963): lemmas 1 and 2]

$$
g(z)=C z^{2} \prod_{n=1}^{\infty}\left(1+\frac{z}{\gamma_{n}}\right)
$$

is an entire function which satisfies

$$
\begin{array}{cc}
\left|g\left(e^{i \theta}\right)\right|<\frac{1}{4}, & 0 \leqq \theta \leqq 2 \pi, \\
\gamma_{n+1}<g\left(\gamma_{n}\right)<2 \gamma_{n+1}, & n=1,2, \cdots, \\
g\left(\gamma_{n}^{1 / 2}\right)<\gamma_{n+1}^{1 / 2}, & n=1,2, \cdots,
\end{array}
$$

and

$$
g\left(\gamma_{n}^{2}\right)>\gamma_{n+1}^{2}, \quad n=1,2, \cdots .
$$

Moreover, if $A_{n}$ denotes the annulus

$$
A_{n}: \gamma_{n}^{2}<|z|<\gamma_{n+1}^{1 / 2},
$$

then by Baker (1963) Theorem 1, there is an integer $N$ such that for all $n>N$ the mapping $z \rightarrow g(z)$ maps $A_{n}$ into $A_{n+1}$, so that $g_{k}(z) \rightarrow \infty$ uniformly in $A_{n}$ as $k \rightarrow \infty$. Since by (3) $g_{k}(z) \rightarrow 0$ uniformly for $|z| \leqq 1$, it is clear that each $\boldsymbol{A}_{n}$, $n>N$, belongs to a multiply connected component $C_{n}$ of $\mathfrak{s}(g)$ and that $C_{n}$ does not meet $\{z:|z| \leqq 1\}$, which belongs to a component of $(5(g)$ which we designate $C_{0}$. It is natural to ask whether the $C_{n}, n>N$, are all different, but this question was left unanswered in Baker (1963). The solution is given by the

THEOREM. For $n>N$ the components $C_{n}$ of $\mathfrak{S}(g)$ described above are all different and each is a wandering domain of $g$.

Proof. Suppose that there are two values of $n>N$ for which $A_{n}$ belong to the same component of $\subseteq(g)$. Suppose $n=m>N$ and $n=m+l, l>0$, are such

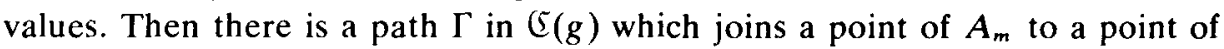
$A_{m+1}$. The path $\Gamma$ must meet $A_{m+1}$, which therefore belongs to the same component of $\mathcal{S}(g)$ as $A_{m}$. So we may take $l=1$. By the complete invariance of $\mathfrak{S}(g)$ the path $g_{k}(\Gamma)$ lies in $(5)(g)$ and it joins $A_{m+k}$ to $A_{m+k+1}, k=1,2, \cdots$. Thus all $A_{n}, n>m$, belong to the same component of $5(g)$, which is therefore multiply-connected and unbounded.

It suffices to show that for all sufficiently large $n$ the annuli $A_{n}$ and $A_{n+2}$ cannot be joined in $\subseteq(g)$. Now for all sufficiently large $n\left(>N_{0}\right)$ we have, since $\gamma_{n} \rightarrow \infty$ in (1) that, 


$$
4 \gamma_{n}^{2}<\gamma_{n+1}^{1 / 2}
$$

Take any $n>\operatorname{Max}\left(\mathrm{N}, \mathrm{N}_{0}\right)$ and assume that $A_{n}, A_{n+2}$ can be joined in $\mathfrak{S}(g)$. Then $z_{1}=2 \gamma_{n}^{2} \in A_{n}$ and $z_{2}=\frac{1}{2} \gamma_{n+3}^{1 / 2} \in A_{n+2}$. There is then a simple polygon joining $z_{1}$ and $z_{2}$ in $\mathscr{S}(g)$ and so $z_{1}, z_{2}$ belong to a simply-connected subdomain, say $H$, of $\widetilde{\varsigma}(g)$. $H$ may be mapped conformally by $z=\psi(t)$ onto $|t|<1$ so that $\psi(0)=z_{1}$ and $\psi(u)=z_{2}$ where $u$ is some value for which $|u|<1$.

Since $g_{k}(z) \rightarrow \infty$ locally uniformly, as $k \rightarrow \infty$ for $z \in A_{n}$, the same is true locally uniformly in the component $G$ of $\left(S(g)\right.$ to which $A_{n}$ belongs. Thus for each integer $p>0, g_{p}(G)$ is a domain in which $G_{k}(z) \rightarrow \infty$ locally uniformly, so $g_{p}(G)$ does not meet the component $G_{0}$ of $(\mathscr{S}(g)$ which includes the disc $\{z:|z| \leqq 1\}$, as $g_{k}(z) \rightarrow 0$ in $G_{0}$. Thus in $G$, and in particular in $H, g(z)$ omits the values 0,1 . Similarly the functions $F_{p}(t)=g_{p}\{\psi(t)\}$ omit the values 0,1 in $|t|<1$. By Schottky's theorem there is a constant $B$, independent of $p$, such that

$$
\begin{aligned}
\left|g_{\rho}\left(z_{2}\right)\right| & =\left|F_{p}(u)\right| \\
& \leqq \exp \left[\left(\frac{1}{1-|u|}\right)\left\{(1+|u|) \log \max \left(1,\left|F_{p}(0)\right|\right)+2 B\right\}\right]
\end{aligned}
$$

Now $g_{p}\left(z_{1}\right)$ is positive and $\rightarrow \infty$ as $p \rightarrow \infty$. so for all sufficiently large $p$ (9) gives, noting $F_{p}(0)=g_{p}\left(z_{1}\right)$,

$$
\left|g_{p}\left(z_{2}\right)\right| \leqq k\left|g_{p}\left(z_{1}\right)\right|^{L}
$$

where $L, K$ are constants which depend on $z_{1}, z_{2}$ but not on $p$. Thus for all sufficiently large $p$ we have

$$
0<g_{p}\left(\frac{1}{2} \gamma_{n+3}^{1 / 2}\right) \leqq K\left\{g_{p}\left(2 \gamma_{n}^{2}\right)\right\}^{L} .
$$

By (8), however, we have

$$
2 \gamma_{n}^{2}<\gamma_{n+1}^{1 / 2}<\gamma_{n+1}
$$

and every iterate $g_{k}$ is positive and increasing on the positive real axis, so for $k \leqq 1$

$$
\begin{aligned}
g_{k}\left(2 \gamma_{n}^{2}\right) & <g_{k}\left(\gamma_{n+1}\right)=g_{k-1}\left\{g\left(\gamma_{n+1}\right)\right\} \\
& <g_{k-1}\left(2 \gamma_{n+2}\right)<g_{k-1}\left(\frac{1}{2} \gamma_{n+3}^{1 / 2},\right.
\end{aligned}
$$

using (4) and (8). For all sufficiently large $x$ one has $g(x)>K x^{L}$ and so for all sufficiently large $k$

$$
g_{k}\left(\frac{1}{2} \gamma_{n+3}^{1 / 2}\right)=g\left\{g_{k-1}\left(\frac{1}{2} \gamma_{n+3}^{1 / 2}\right)\right\}>g\left\{g_{k}\left(2 \gamma_{n}^{2}\right)\right\}>K\left\{g_{k}\left(2 \gamma_{n}^{2}\right)^{L}\right\},
$$

which contradicts (10). Thus the first assertion of the theorem is established: for $n>N$ the components $C_{n}$ of $5(g)$ which contain $A_{n}$ are all different, and each is a bounded domain. 
It follows at once that each $C_{n}$ is a wandering domain for $g$. If this is not the case, then there exist integers $n>N, k>0, l>0$ such that $g_{k}\left(C_{n}\right)$ meets $g_{k+1}\left(C_{n}\right)$, i.e. since $g_{k}\left(C_{n}\right) \subset C_{n+k}, g_{l}\left(G^{\prime}\right) \subset G^{\prime}$, where $G^{\prime}=C_{n+k}$. The sequence $\left\{g_{i n}(z)\right\}, n=1,2, \cdots$ is bounded in $G^{\prime}$, taking values only in $G^{\prime}$. But this contradicts the fact that the whole sequence $\left\{g_{k}\right\}, k=1,2, \cdots$, tends locally uniformly to $\infty$ in $G^{\prime}$, as in every $C_{n}, n>N$.

The theorem is now established and clears up the problem of the existence of wandering domains, at least in the case of entire functions. It adds a little to the discussion of Baker (1963) where it was shown that, if for entire $g$ the set $\mathfrak{E}(\mathrm{g})$ has a multiply-connected component, $G$, then there are just two alternatives, namely:

I. $G$ is unbounded and completely invariant and every other component of $\mathfrak{E}(f)$ is simply-connected, or

II. All components of $\mathscr{E}(f)$ are bounded and infinitely many of them are multiply-connected.

It was conjectured in Baker (1963) that alternative II occurred in the case of the $g$ of our theorem and this is now established. It is interesting to note [c.f Baker (1963)] that truncating the infinite product in (2) gives a polynomial

$$
P(z)=C z^{2} \prod_{n=1}^{k}\left(1+\frac{z}{\gamma_{n}}\right)
$$

such that alternative I applies to $(E(P)$ which has an unbounded and multiplyconnected component.

\section{References}

I. N. Baker (1963), 'Multiply connected domains of normality in iteration theory', Math. Zeitschrift $81,206-214$.

P. Fatou (1919) (1920), 'Sur les équations fonctionelles', Bull. Soc. Mash. France 47, $161-271$ and $48,33-94,208-314$.

P. Fatou (1926), 'Sur l'itération des fonctions transcendantes entières', Acta Math. 47, 337-370.

M. V. Jacobson (1969) 'On the problem of the classification of polynomial endomorphisms of the plane', Math U.S.S.R. Sbornik 9, 345-364 = Engl. transl. of Mat. Sbornik 80, (122) No. 3 (Russian).

G. Julia (1918), 'Mémoire sur l'itération des fonctions rationelles', J. de Math. Pures et Appliquées (8) I, 47-245.

D. A. Pelles (1974), Iteration of endomorphisms of $P^{n}(C)$, Univ. of North Carolina (mimeographed).

Department of Mathematics

Imperial College of Science \& Technology

London, SW7. 2RH

England 